\title{
Anisotropic Standard Decelerating Cosmological Model with Quadratic Equation of State
}

\author{
Mohammad Moksud Alam, Mohammed Aman Ullah*, S M Erfanul Kabir Chowdhury \\ Department of Mathematics, University of Chittagong, Chittagong, Bangladesh \\ Email: moksud.math@cu.ac.bd, ^aman@cu.ac.bd, erfancu@gmail.com
}

How to cite this paper: Alam, M.M., Ullah, M.A. and Chowdhury, SMEK. (2020) Anisotropic Standard Decelerating Cosmological Model with Quadratic Equation of State. Journal of Applied Mathematics and Physics, 8, 1990-1998.

https://doi.org/10.4236/jamp.2020.89148

Received: July 22, 2020

Accepted: September 25, 2020

Published: September 28, 2020

Copyright $\odot 2020$ by author(s) and Scientific Research Publishing Inc. This work is licensed under the Creative Commons Attribution International License (CC BY 4.0).

http://creativecommons.org/licenses/by/4.0/

\begin{abstract}
Spatially homogeneous and anisotropic Bianchi type-I cosmological model containing perfect fluid with quadratic equation of state has been diagnosed in general theory of relativity. To obtain a deterministic solution, we have used a relation between metric potentials. The exact solution of Einstein's field equations thus obtained represents an expanding and decelerating universe. The physical and kinematical parameters of the model have also been analyzed with certain constrained between the parameters of the quadratic equation of state.
\end{abstract}

\section{Keywords}

General Relativity, Bianchi Type-I, Cosmological Model, Quadratic Equation of State (EoS)

\section{Introduction}

In a large scale structure, the universe is highly homogeneous and isotropic, shown in [1]. These properties are also supported by the work of Benet et al. [2] and Spergel et al. [3] via the cosmic microwave background radiation. Aside homogeneity property, isotropy throws a challenge that anisotropic models cannot be isotropized appropriately because of their time evolvement in future. However this is to note that inflation is a suitable method to solve this problem.

Ananda and Bruni's [4] works in relativistic dynamics study of Robertson Walker models using a non-linear quadratic equation of state (EoS) are excellent to enhance to open new door of research. The authors considered an equation of state as of the form $p=p_{0}+\alpha \rho+\beta \rho^{2}$ (where, $p_{0}, \alpha$ and $\beta$ are parameters), ${ }^{*}$ Corresponding author. 
in particular the quadratic term in the above equation and revealed that anisotropy at the singularity existing in brane world scenario can be reproduced in the context of general relativity.

Depending on the appearance or look of the different forms of quadratic EoS, there are extensive uses of it in many contemporary research areas. For instances, EoS of the form $p=\alpha \rho+\left(\rho^{2} / \rho_{c}\right)$ has been introduced by Ananda and Bruni [5] to study the effect of quadratic EoS in homogeneous and inhomogeneous cosmological models to isotropize the universe at early age when initial singularity is continued.

In a firm study, Nojiri and Odintsov [6] used inhomogeneous Hubble parameter term to discuss the variation of dark energy universe relative to different equation of state. In 2006, Capozzelio [7] pointed out that the observational constraint impact along with quadratic EoS on dark energy. Nojiri et al. [6] works reveal that quadratic EoS may be nice tool to describe dark energy or unified dark matter in a more generous way. In 2009, Rahaman et al. [8] showed how electron can be modeled precisely instead of spherically symmetric charged perfect fluid distribution of matter characterized by quadratic EoS. Followed by the works of Rahaman et al. [8], Firoze et al. [9] introduced "Charged anisotropic matter" into quadratic EoS where the approach results in a new class of static spherically symmetric models of relativistic star. Einstein and Maxwell equations were brought under the application of quadratic EoS. For charged anisotropic matter distribution, Maharaj and Takisa [10] found a new exact solution of Einstein-Maxwell equation in terms of quadratic EoS. Unification of vacuum, dark energy and radiation [11] was also brought under active consideration through a cosmological model based on quadratic EoS.

Later Chavanis [12] considered early inflation, intermediate decelerating expansion, late accelerating expansion as quadratic EoS and developed a model. In 2013, Sharma and Ratanpal's work [13] results in a favorable solution focusing the interior of a static symmetric spherically asymmetric compact anisotropic star and showed that the model admits EoS in quadratic form. After Maharaj and Tasika [10], Malaver [14] solved Maxwell in Maxwell system of equations using some elementary function throughout the behavior of compact anisotropic relativistic objects in quadratic equation of state.

It reveals from the above discussion that quadratic EoS plays an important and interesting role in many areas including dark energy and dynamics of different models in general relativity. In the study of anisotropic problem, in particular, Bianchi type-I homogeneous cosmological model containing perfect fluid is an interesting idea that lead us to continue research using quadratic EoS to generate an expanding and decelerating universe.

We have organized this paper as follows: In Section 2, we have applied Bianchi type-I metric to obtain field equations. Explicit solution of the equations is presented in Section 3 followed by relevant interpretations of the results in Section 4. We summarize this paper by giving a conclusion in Section 5 . 


\section{Metric and Field Equations}

The Bianchi type-I line element is given by

$$
\mathrm{ds} s^{2}=\mathrm{d} t^{2}-A^{2} \mathrm{~d} x^{2}-B^{2} \mathrm{~d} y^{2}-C^{2} \mathrm{dz} z^{2}
$$

where $A, B$ and $C$ are scale factors and are functions of time $t$ only.

The Einstein field equations, in natural limits ( $8 \pi G=1$ and $c=1)$, are

$$
R_{i j}-\frac{1}{2} R g_{i j}=-T_{i j}
$$

where $R_{i j}$ is the Ricci tensor, $R$ is the Ricci scalar and $T_{i j}$ is the energy-momentum tensor.

The energy-momentum tensor $T_{i j}$ for the perfect fluid is given by

$$
T_{i j}=(\rho+p) u_{i} u_{j}-p g_{i j}
$$

where $\rho$ is the energy density, $p$ is the pressure and $u^{i}$ is the four velocity vector satisfying $g_{i j} u^{i} u_{j}=1$.

Here, we have assumed an equation of state (EoS) in the general form $p=p(\rho)$ for the matter distribution.

We have considered it in the quadratic form as follows

$$
p=\alpha \rho^{2}+\beta \rho+\gamma
$$

where $\alpha, \beta$ and $\gamma$ are the constant and strictly $\alpha \neq 0$ which preserves the quadratic nature of the equation of state.

In co-moving coordinate system, the Einstein field Equations (2) for the metric (1) with the help of Equation (3) reduce to following set of equations:

$$
\begin{aligned}
& \frac{\dot{A} \dot{B}}{A B}+\frac{\dot{B} \dot{C}}{B C}+\frac{\dot{A} \dot{C}}{A C}=\rho \\
& \frac{\ddot{B}}{B}+\frac{\ddot{C}}{C}+\frac{\dot{B} \dot{C}}{B C}=-p \\
& \frac{\ddot{A}}{A}+\frac{\ddot{C}}{C}+\frac{\dot{A} \dot{C}}{A C}=-p \\
& \frac{\ddot{A}}{A}+\frac{\ddot{B}}{B}+\frac{\dot{A} \dot{B}}{B C}=-p
\end{aligned}
$$

where overhead dot $(\cdot)$ denotes differentiation with respect to time $t$.

The vanishing divergence of Einstein tensor which leads to $T_{; j}^{i j}=0$, yields the following energy conservation equation

$$
\dot{\rho}+\left(\frac{\dot{A}}{A}+\frac{\dot{B}}{B}+\frac{\dot{C}}{C}\right)(\rho+p)=0
$$

We have defined the spatial volume $V$ and average scale factor " $a$ " for Bianchi type-I space-time as

$$
V=a^{3}=A B C
$$

The mean Hubble parameter $H$ for Bianchi type-I universe is defined as 


$$
H=\frac{\dot{a}}{a}=\frac{1}{3}\left(H_{x}+H_{y}+H_{z}\right)
$$

where $H_{x}=\frac{\dot{A}}{A}, H_{y}=\frac{\dot{B}}{B}, H_{z}=\frac{\dot{C}}{C}$ are the directional Hubble parameters in the directions of $x, y$ and $z$ axes respectively.

\section{Solution of the Field Equations}

The field Equation (5)-(8) are four independent equations in five unknowns $A$, $B, C, \rho$ and $p$. For the exact explicit solutions to these Einstein's field equations, we have assumed the expansion scalar $(\theta)$ as proportional to the component $\sigma_{1}^{1}$ of the shear tensor $\sigma_{i}^{j}$ in the model that leads to

$$
A=(B C)^{m}
$$

Subtracting (7) from (8), we have

$$
\frac{\ddot{B}}{B}-\frac{\ddot{C}}{C}+\frac{\dot{A}}{A}\left(\frac{\dot{B}}{B}-\frac{\dot{C}}{C}\right)=0
$$

From equations (12) and (13), we get

$$
\frac{\ddot{B}}{B}-\frac{\ddot{C}}{C}+m\left(\frac{\dot{B}}{B}-\frac{\dot{C}}{C}\right)\left(\frac{\dot{B}}{B}+\frac{\dot{C}}{C}\right)=0
$$

which can be reduced to the following form

$$
\frac{\frac{\mathrm{d}}{\mathrm{d} t}\left(\frac{\dot{B}}{B}-\frac{\dot{C}}{C}\right)}{\frac{\dot{B}}{B}-\frac{\dot{C}}{C}}+(m+1)\left(\frac{\dot{B}}{B}+\frac{\dot{C}}{C}\right)=0
$$

Integrating Equation (15), we obtain

$$
\left(\frac{\dot{B}}{B}-\frac{\dot{C}}{C}\right)(B C)^{m+1}=l_{1}
$$

Equation (16) can be written as follows

$$
\frac{\mathrm{d}}{\mathrm{d} t}\left(B^{m+1}\right)-(m+1) \frac{\dot{C}}{C} B^{m+1}=(m+1) \frac{l_{1}}{C^{m+1}}
$$

which is a first order linear differential equation in $B^{m+1}$ and $t$. Hence the general solution of Equation (17) is found as

$$
B^{m+1}=(m+1) C^{m+1} \int \frac{l_{1}}{C^{2(m+1)}} \mathrm{d} t+l_{2} C^{m+1}
$$

where $l_{1}$ and $l_{2}$ are integrating constants.

It is to be noted that in this case the solution of Einstein's field equations reduces to the integration of (18) if the explicit form of the scale factor $C$ is known.

We obtain particular solution of (18) for a simple choice of the function $C$. For this, we choose,

$$
C=t^{n}
$$


where $n$ is real number. Then (18) yields

$$
B^{m+1}=\frac{(m+1) l_{1}}{1-2(m+1) n} t^{1-(m+1) n}+l_{2} t^{(m+1) n}
$$

Choosing $l_{2}=0$ without loss of generality and hence we obtain

$$
B=\left\{\frac{(m+1) l_{1}}{1-2(m+1) n}\right\}^{\frac{1}{m+1}} t^{\frac{1}{m+1}-n}
$$

From Equation (12), (19) and (21), we get

$$
A=\left\{\frac{(m+1) l_{1}}{1-2(m+1) n}\right\}^{\frac{m}{m+1}} t^{\frac{m}{m+1}}
$$

The metric of the model can be written in the form

$\mathrm{d} s^{2}=\mathrm{d} t^{2}-\left\{\frac{(m+1) l_{1}}{1-2(m+1) n}\right\}^{\frac{2 m}{m+1}} t^{\frac{2 m}{m+1}} \mathrm{~d} x^{2}-\left\{\frac{(m+1) l_{1}}{1-2(m+1) n}\right\}^{\frac{2}{m+1}} t^{\frac{2}{m+1}-2 n} \mathrm{~d} y^{2}-t^{2 n} \mathrm{~d} z^{2}$

Using the directional scale factors for the model (23) in (10), we compute the spatial volume $V$ as follows

$$
V=\frac{(m+1) l_{1}}{1-2(m+1) n} t
$$

For the spatial volume to be positive, we must have $\frac{(m+1) l_{1}}{1-2(m+1) n} t>0$. It is seen that the spatial volume is zero at $t=0$.

Choosing $\beta=1$ and $\gamma=0$ in Equation (4) as studied in [15] [16] [17] and from Equations (5) and (6) by using Equations (19), (21) and (22), we obtain the energy density for the model (23) as

$$
\rho=\sqrt{\frac{1}{\alpha}\left\{-\frac{n+1}{m+1}-(m-n)^{2}\right\}} \frac{1}{t}
$$

From (4) and (25), we obtain the pressure as

$$
p=\left\{-\frac{n+1}{m+1}-(m-n)^{2}\right\} \frac{1}{t^{2}}+\sqrt{\frac{1}{\alpha}\left\{-\frac{n+1}{m+1}-(m-n)^{2}\right\}} \frac{1}{t}
$$

Again, choosing $\beta=-1$ and $\gamma=0$ in Equation (4) as in [18] and from Equations (5) and (6) by using Equations (19), (21) and (22), we obtain the energy density for the model (23) as

$$
\begin{aligned}
\rho= & \sqrt{\frac{1}{\alpha}\left\{\frac{m-1}{(m+1)^{2}}+\frac{m n-m^{2}+2 m+1}{m+1}-m(m+1)-n(n-1)\right\}} \frac{1}{t} \\
p= & \left\{\frac{m-1}{(m+1)^{2}}+\frac{m n-m^{2}+2 m+1}{m+1}-m(m+1)-n(n-1)\right\} \frac{1}{t^{2}} \\
& -\sqrt{\frac{1}{\alpha}\left\{\frac{m-1}{(m+1)^{2}}+\frac{m n-m^{2}+2 m+1}{m+1}-m(m+1)-n(n-1)\right\}} \frac{1}{t}
\end{aligned}
$$


Some other physically important parameters for the model (23) such as the mean Hubble parameter $H$, the scalar expansion $\theta$, the average anisotropy parameter $\Delta$, the shear scalar $\sigma^{2}$ and deceleration parameter $q$ which are defined and found to be

$$
\begin{gathered}
H=\frac{1}{3}\left(\frac{\dot{A}}{A}+\frac{\dot{B}}{B}+\frac{\dot{C}}{C}\right)=\frac{1}{3 t} \\
\theta=3 H=\frac{1}{t} \\
\Delta=\frac{1}{3} \sum_{i=1}^{3}\left(\frac{\Delta H_{i}}{H}\right)^{2}=\frac{2\left(m^{2}-m+1\right)+6 n(m+1)(m n+n-1)}{(m+1)^{2}} \\
\sigma^{2}=\frac{3}{2} \Delta H^{2}=\frac{\left(m^{2}-m+1\right)+3 n(m+1)(m n+n-1)}{3(m+1)^{2} t^{2}} \\
q=\frac{\mathrm{d}}{\mathrm{d} t}\left(\frac{1}{H}\right)-1=2
\end{gathered}
$$

\section{Results and Discussions}

It has been observed from Equation (25) and Figure 1 that the energy density $\rho$ is a decreasing function of time and $\rho>0$ while diverges at the initial epoch of the cosmological evolution for $\alpha=-1$ and $\beta=1$. Also, from Equation (27) and Figure 1, it is clearly seen that the energy density $\rho$ is a decreasing function of time and $\rho>0$ while diverges at the initial epoch of the cosmological evolution for $\alpha=1$ and $\beta=-1$. Moreover, we have investigated through using Matlab tools that it is obvious to require a fine tuning between $\alpha$ and $\beta$ to obtain physically viable cosmological model [15] [16] [17] [18]. In an agreement with the energy dominated universe, the model, with $\alpha=-1$ and $\beta=1$, shows a negative pressure corresponding to Figure 2 and Equation (26).

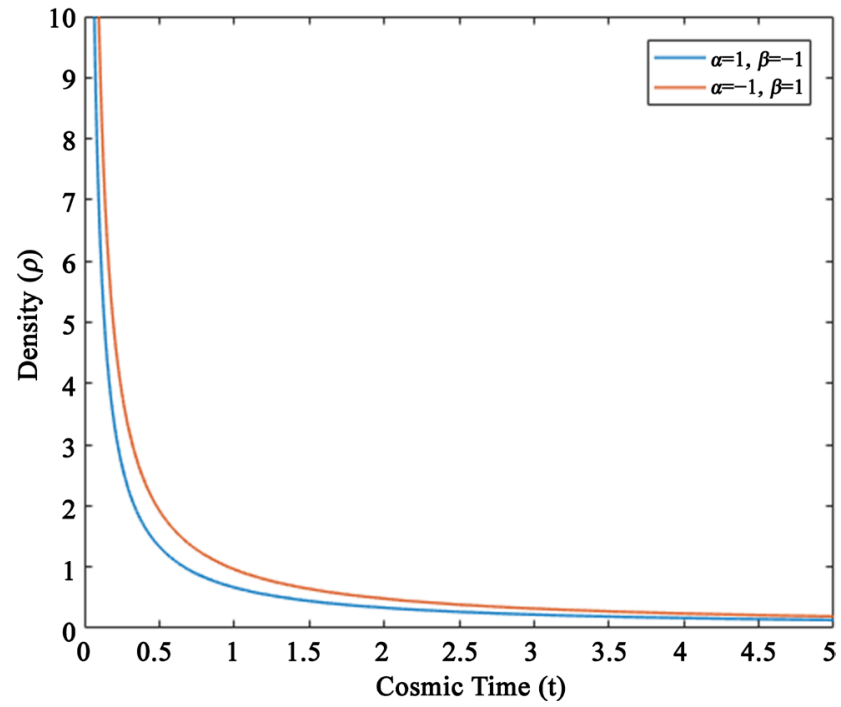

Figure 1. Density vs Cosmic Time using Equations (25) and (27) $(m=0.2, n=0.1)$. 


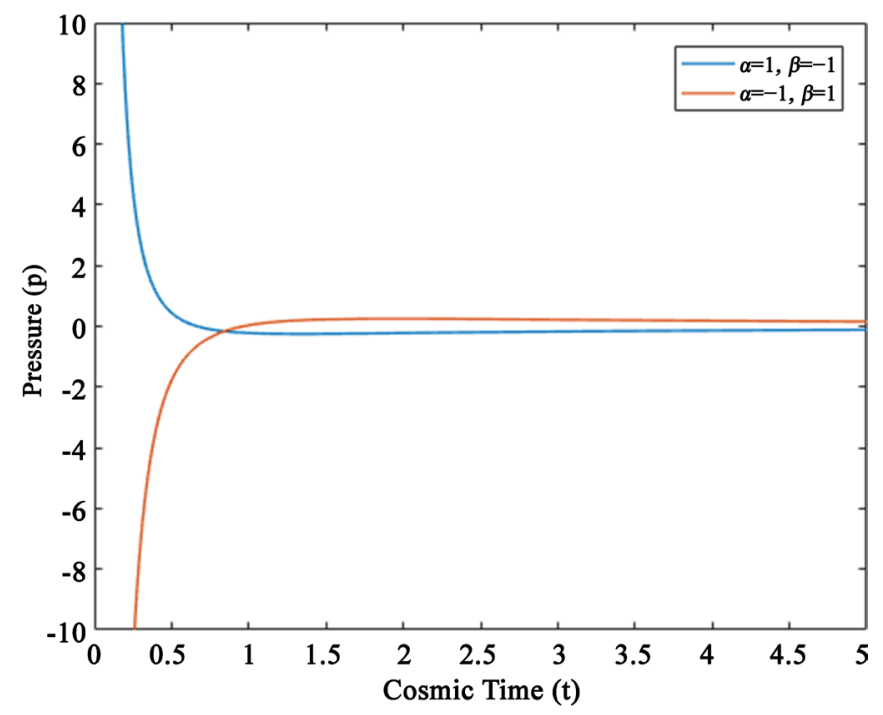

Figure 2. Pressure vs Cosmic Time using Equations (26) and (28) $(m=0.2, n=0.1)$.

Again, Figure 2, corresponding to Equation (28) for $\alpha=1$ and $\beta=-1$, depicts the evolution of pressure $p$ and shows that $p$ is positive and $p \rightarrow 0$ when $t \rightarrow \infty$ as in matter dominated universe.

From Equation (24), it has been observed that initially, the spatial volume $V$ vanishes and linearly increases as time $t$ increases and becomes infinitely large for $t \rightarrow \infty$. This shows that the universe starts expanding with initial zero volume and keeps expanding with increase of cosmic time $t$. From Equation (29), it has been observed that Hubble parameter is a decreasing function of time and $H \rightarrow 0$ when $t \rightarrow \infty$. The expansion scalar and shear scalar start with infinitely large value at $t=0$ and tend to zero as $t \rightarrow \infty$ corresponding to the Equations (30) and (32) which confirms the expansion of the universe. The mean anisotropy parameter, as in Equation (31), is independent of time and is uniform throughout the whole expansion of the universe when $m \neq-1$, but for $m=-1$, it tends to infinity. Also, since $\left(\sigma^{2} / \theta^{2}\right)=$ constant except at $m \neq-1$, the model does not approach to isotropy.

The deceleration parameter $(q)$ is an important parameter to determine whether the model inflates or not. The negative value of $q$ confirms inflation whereas the positive value of $q$ corresponds to standard deceleration. Our model shows decelerating behavior as the deceleration parameter is a positive constant. The remarkable thing is that though the recent observations of SNeIa [19] [20] as well as CMBR [2] [3] are in favour of accelerating models $(q<0)$, but both do not entirely rule out the decelerating ones which are also realistic with these observations supported by the work of Vishwakarma [21]. Moreover, the study of the decelerating model of the universe is important to make a bridge between early inflation and late time acceleration.

\section{Conclusion}

We investigated spatially homogeneous and anisotropic Bianchi type-I cosmo- 
logical models with quadratic equation of state (EoS) in the context of general relativity. The important physical and kinematical parameters in the discussion of cosmological model have been determined and studied. The limitation in the choices of the parameters of quadratic equation of state has been explored and discussed. We also showed, the universe will not be shear free and will remain anisotropic throughout the cosmic evolution with standard deceleration.

\section{Conflicts of Interest}

The authors declare no conflicts of interest regarding the publication of this paper.

\section{References}

[1] Tegmark, M., et al. (2004) Cosmological Parameters from SDSS and WMAP. Physical Review D, 69, Article ID: 103501.

[2] Bennett, C.L., et al. (2003) First-Year Wilkinson Microwave Anisotropy Probe (WMAP) Observations: Preliminary Maps and Basic Results. Astrophysical Journal Supplement Series, 148, 1-27. https://doi.org/10.1086/377253

[3] Spergel, D.N., et al. (2003) First-Year Wilkinson Microwave Anisotropy Probe (WMAP)* Observations: Determination of Cosmological Parameters. The Astrophysical Journal Supplement Series, 148, 175-194. https://doi.org/10.1086/377226

[4] Ananda, K. and Bruni, M. (2005) Cosmological Dynamics and Dark Energy with a Non-Linear Equation of State: A Quadratic Model. https://doi.org/10.1103/PhysRevD.74.023523

[5] Ananda, K.N. and Bruni, M. (2006) Cosmo-Dynamics and Dark Energy with a Quadratic EoS: Anisotropic Models, Large-Scale Perturbations and Cosmological Singularities. Physical Review D, 74, Article ID: 023523. https://doi.org/10.1103/PhysRevD.74.023524

[6] Nojiri, S. and Odintsov, S.D. (2005) Inhomogeneous Equation of State of the Universe: Phantom Era, Future Singularity, and Crossing the Phantom Barrier. Physical Review D, 72, Article ID: 023003. https://doi.org/10.1103/PhysRevD.72.023003

[7] Capozziello, S., Cardone, V.F., Elizalde, E., Nojiri, S. and Odintsov, S.D. (2006) Observational Constraints on Dark Energy with Generalized Equations of State. Physical Review D, 73, Article ID: 043512. https://doi.org/10.1103/PhysRevD.73.043512

[8] Rahaman, F., Jamil, M. and Chakraborty, K. (2010) Revisiting the Classical Electron Model in General Relativity. Astrophysics and Space Science, 331, 191-197. https://doi.org/10.1007/s10509-010-0446-3

[9] Feroze, T. and Siddiqui, A.A. (2011) Charged Anisotropic Matter with Quadratic Equation of State. General Relativity and Gravitation, 43, 1025-1035. https://doi.org/10.1007/s10714-010-1121-2

[10] Maharaj, S.D. and Takisa, P.M. (2012) Regular Models with Quadratic Equation of State. General Relativity and Gravitation, 44, 1419-1432. https://doi.org/10.1007/s10714-012-1347-2

[11] Chavanis, P.H. (2013) A Cosmological Model Based on a Quadratic Equation of State Unifying Vacuum Energy, Radiation, and Dark Energy. Journal of Gravity, 2013, Article ID: 682451. https://doi.org/10.1155/2013/682451

[12] Chavanis, P.H. (2015) A Cosmological Model Describing the Early Inflation, the Intermediate Decelerating Expansion, and the Late Accelerating Expansion of the 
Universe by a Quadratic Equation of State. Universe, 1, 357. https://doi.org/10.3390/universe1030357

[13] Sharma, R. and Ratanpal, B.S. (2013) Relativistic Stellar Model Admitting a Quadratic Equation of State. International Journal of Modern Physics D, 22, Article ID: 1350074. https://doi.org/10.1142/S0218271813500740

[14] Malaver, M. (2014) Strange Quark Star Model with Quadratic Equation of State. Frontiers of Mathematics and Its Applications, 1, 9-15.

[15] Rahaman, F., et al. (2009) Revisiting the Classical Electron Model in General Relativity.

[16] Tiwari, R.K., et al. (2017) Time Varying $G$ and $\Lambda$ Cosmology in $f(R, T)$ Gravity Theory. Astrophysics and Space Science, 362, 143. https://doi.org/10.1007/s10509-017-3111-2

[17] Ahmed, N., et al. (2019) Transition from Decelerating to Accelerating Universe with Quadratic Equation of State in $f(R, T)$ Gravity. NRIAG Journal of Astronomy and Geophysics, 8, 198-203. https://doi.org/10.1080/20909977.2019.1668116

[18] Reddy, D.R.K., Adhav, K.S. and Purandare, M.A. (2015) Bianchi Type-I Cosmological Model with Quadratic Equation of State. Astrophysics and Space Science, 357, 20. https://doi.org/10.1007/s10509-015-2302-y

[19] Riess, A.G., et al. (1998) Observational Evidence from Supernovae for an Accelerating Universe and a Cosmological Constant. The Astronomical Journal, 116, 1009-1038. https://doi.org/10.1086/300499

[20] Perlmutter, S., et al. (1999) Measurements of $\Omega$ and $\Lambda$ from 42 High-Redshift Supernovae. The Astronomical Journal, 517, 565. https://doi.org/10.1086/307221

[21] Vishwakarma, R.G. (2000) A Study of Angular Size-Redshift Relation for Models in Which $\Lambda$ Decays as the Energy Density. Classical and Quantum Gravity, 17, 3833. https://doi.org/10.1088/0264-9381/17/18/317 